\title{
THE ACS SURVEY OF GLOBULAR CLUSTERS. XIII. PHOTOMETRIC CALIBRATION IN COMPARISON WITH STETSON STANDARDS*
}

\author{
Maren Hempel ${ }^{1,2,14}$, Ata Sarajedini ${ }^{2}$, Jay Anderson $^{3}$, Antonio Aparicio $^{4}$, Luigi R. Bedin $^{5}$, Brian Chaboyer $^{6}$, \\ Steven R. Majewski ${ }^{7}$, Antonio Marín-Franch ${ }^{8}$, Antonino Milone ${ }^{9}$, Nathaniel E. Q. Paust ${ }^{10}$, \\ Giampaolo Piotto $^{11}$, I. Neill Reid ${ }^{3}$, Alfred Rosenberg ${ }^{12}$, and Michael Siegel ${ }^{13}$ \\ ${ }^{1}$ Instituto de Astrofísica, Facultad de Fisica, Pontificia Universidad Católica de Chile, \\ Vicuña Mackenna 4860, 7820436 Macul, Santiago, Chile; mhempel@ astro.puc.cl \\ 2 Department of Astronomy, University of Florida, 211 Bryant Space Science Center, Gainesville, FL 32611, USA; ata@ astro.ufl.edu \\ ${ }^{3}$ Space Telescope Science Institute, 3700 San Martin Drive, Baltimore, MD 21218, USA; jayander@ stsci.edu, inr@ stsci.edu \\ ${ }^{4}$ Department of Astrophysics, University of La Laguna, Vía Láctea s/n, E-38200 La Laguna, Spain; antapaj@iac.es \\ ${ }^{5}$ INAF/Osservatorio Astronomico di Padova, Vicolo dell'Osservatorio 5, I-35122 Padova, Italy; luigi.bedin@ oapd.inaf.it \\ ${ }^{6}$ Department of Physics and Astronomy, Dartmouth College, 6127 Wilder Laboratory, Hanover, NH 03755, USA; Brian.Chaboyer@ dartmouth.edu \\ ${ }^{7}$ Department of Astronomy, University of Virginia, P.O. Box 400325, Charlottesville, VA 22904-4325, USA; srm4n@ virginia.edu \\ ${ }^{8}$ Centro de Estudios de Física del Cosmos de Aragón, Plaza. San Juan, 1 Planta-2, E-44001 Teruel, Spain; amarin@cefca.es \\ ${ }^{9}$ College of Physical and Mathematical Sciences, Australian National University, Canberra, ACT 0200, Australia; antonino.milone@ anu.edu.au \\ ${ }^{10}$ Whitman College, 345 Boyer Avenue, Walla Walla, WA 99362, USA; paustne@ whitman.edu \\ ${ }^{11}$ Dipartimento di Astronomia, University di Padova, vicolo dell’Osservatorio 5, I-35122 Padova, Italy; giampaolo.piotto@ unipd.it \\ ${ }^{12}$ Instituto de Astrofísica de Canarias, Vía Láctea s/n, E-38200 La Laguna, Spain; alf@iac.es \\ ${ }^{13}$ Department of Astronomy and Astrophysics, Pennsylvania State University, 525 Davey Laboratory, State College, PA 16801, USA; siegel@ swift.psu.edu \\ Received 2013 October 8; accepted 2013 November 29; published 2014 January 31
}

\begin{abstract}
In this study we compare the photometric data of 34 Milky Way globular clusters, observed within the Advanced Camera for Surveys (ACS) Treasury Program (PI: A. Sarajedini) with the corresponding ground-based data, provided by the Photometric Standard Field Catalogs of Stetson. We focus on the transformation between the Hubble Space Telescope/ACS F606W to V-band and F814W to I-band only. The goal is to assess the validity of the filter transformation equations by Sirianni et al. with respect to their dependence on metallicity, horizontal branch morphology, mass, and integrated $(V-I)$ color of the various globular clusters. The transformation equations as recommended by Sirianni et al. are based on synthetic photometry, were mostly tested on NGC 2419, and may introduce additional uncertainties when applied to different stellar populations. Such a dependence is expected due to the fact that the transformation equations are based on the observations of only one globular cluster, i.e., NGC 2419. Surprisingly, the correlation between offset and metallicity is found to be weak, with a low level significance. The correlation between offset and horizontal branch structure, as well as total cluster mass is still weaker. Based on the available data we do not find the photometric offset to be linked to multiple stellar populations, e.g., as found in NGC 0288, NGC 1851, and NGC 5139. The results of this study show that there are small systematic offsets between the transformed ACS- and observed ground-based photometry, and that these are only weakly correlated, if at all, with various cluster parameters and their underlying stellar populations. As a result, investigators wishing to transform globular cluster photometry from the Sirianni et al. ground-based $V, I$ system onto the Stetson system simply need to add $-0.040( \pm 0.012)$ to the $V$ magnitudes and $-0.047( \pm 0.011)$ to the $I$ magnitudes. This in turn means that the transformed ACS $V-I$ colors match the ground-based values from Stetson to within $\sim 0.01 \mathrm{mag}$.
\end{abstract}

Key words: globular clusters: general - stars: imaging - techniques: miscellaneous

Online-only material: color figures

\section{INTRODUCTION}

The Advanced Camera for Surveys (ACS) on board the Hubble Space Telescope (Pavlovsky et al. 2005) is one of the cornerstones of astronomical research. In particular, its high spatial resolution combined with its Wide Field Channel make it exceptionally well suited to investigating dense stellar systems like globular clusters (GCs), not only in our own Milky Way (e.g., Sarajedini et al. 2007), but also in extragalactic systems (e.g., Côté et al. 2004; Jordán et al. 2007). The results of these studies are often matched with independent data obtained with ground-based instruments to extend the field of view (FOV) or

\footnotetext{
* Based on observations made with the NASA/ESA Hubble Space Telescope, obtained at the Space Telescope Science Institute, which is operated by AURA, Inc., under NASA contract NAS 5-26555, under program GO-10775 (PI: A. Sarajedini).

${ }^{14}$ Also at The Milky Way Millennium Nucleus, Av. Vicuña Mackenna 4860, 782-0436 Macul, Santiago, Chile.
}

the wavelength range of the observations. However, it is important to note that the ACS filters differ significantly from ground-based filter sets and that the photometric transformation between them could depend on knowledge of the underlying stellar population, e.g., the age or metallicity of the object being observed. In addition, these photometric data, resolved and integrated, are used to derive the metallicity and other parameters of stars and stellar populations. Clearly, it is paramount to estimate the accuracy of the photometric transformation procedure.

The recommended standard ACS to ground-based photometric transformation equations, as described by Sirianni et al. (2005), are based on synthetic photometry in contrast to using observational data. The accuracy was tested by a comparison of the transformed ACS photometry in several bands with the Stetson standard field data in NGC 2419 (e.g., Stetson 2000, 2005), a massive GC in the Milky Way (Stetson 2000, 2005; Baumgardt et al. 2009). The Stetson data offer ground-based Johnson/Kron-Cousins $U-, B-, V-, R$-, and 
I-band photometry of $>1300$ stars. With a metallicity of $[\mathrm{Fe} / \mathrm{H}]=-2.14$ (Zinn 1985; Suntzeff et al. 1988) and total luminosity of $M_{V}=-9.42$ (Harris 1996, 2010 edition, ${ }^{15}$ ), NGC 2419 is not only one of the most metal-poor, but also the fourth brightest known Milky Way globular cluster (MWGC). As recently discovered by Di Criscienzo et al. (2011), NGC 2419 contains "a large and extreme" second stellar generation, representing $\sim 30 \%$ of the total stellar population, and featuring different He content.

The Milky Way globular clusters vary in many ways, i.e., by metallicity, color, age, or $\alpha$-enhancement. Therefore we need to determine whether the accuracy of the transformed luminosities depends in any way on those cluster parameters, i.e., whether the transformation between various filter systems introduces additional, and more importantly, cluster-dependent uncertainties. In a similar study, Saha et al. (2005) used NGC 2419, Palomar 4 and Palomar 14 and a set of standard star observations to extend the validity range of the HST/ WFPC2 calibration to stars as faint as $V \approx 21 \mathrm{mag}$. These differences, which we will henceforth call the "photometric offset" or short "offset," are the subject of this study. Here we compare the transformed $V$ - and $I$-band photometry of various MWGCs, derived from ACS F606W and F814W observations, with their counterparts by Stetson $(2000,2005)$, i.e., Johnson $V$ and Kron-Cousins $I$, respectively (which we will be calling $V$ and $I$ throughout this paper). When referring to the ACS based data, we will use the term "transformed" $V$ and $I$. Our goal is to search for, and if confirmed, to quantify the correlation between the photometric offset and various cluster parameters.

The outline of this paper is as follows. In Section 2 we briefly describe the ACS and Stetson data set, and, in more detail, discuss how both photometric catalogs were matched. Section 3 focuses on the photometric offsets and their correlation with the GC parameters, such as integrated color, metallicity, and horizontal branch (HB) structure. Given that HB structure and metallicity are closely linked, we will investigate the correlation between photometric offset, metallicity and $\mathrm{HB}$ structure separately for the HB stars only. Due to the mounting evidence that more massive GCs tend to harbor multiple stellar populations (e.g., Piotto 2009; Milone et al. 2010; Roh et al. 2011), we include the total cluster mass in the list of cluster parameters and discuss the effect of NGC 5139, the most massive target cluster, which also known to host multiple stellar populations (e.g., Lee et al. 1999; Pancino et al. 2000; Bedin et al. 2004; Da Costa et al. 2009; Bellini et al. 2010). Given the wide spread of GC parameters, Section 4 deals with the effects this diversity may have on the standard deviation of the photometric offset, and therefore the accuracy of the derived correlation coefficients. We summarize our findings in Section 5.

\section{PHOTOMETRIC SAMPLE AND MATCHING PROCEDURE}

This study is based on the photometric catalogs for 34 MWGCs observed in the ACS Survey of Galactic Globular Clusters (PI: A. Sarajedini; see Sarajedini et al. 2007) and the Photometric Standard Field Catalog by P. Stetson. ${ }^{16}$ Detailed information on the data reduction applied to both samples can be found in Sarajedini et al. (2007) and Anderson et al. (2008) for the ACS survey and Stetson (2000, 2005), respectively. The purpose of this study is to compare the converted $V$ - and $I$-band

\footnotetext{
15 http://physwww.mcmaster.ca/ harris/mwgc.dat.

16 http://www4.cadc.hia.nrc.gc.ca/community/STETSON/standards.
}

photometry of the ACS observations with their counterparts from Stetson and hence particular care has to be taken when matching the photometry of individual stars, given the high stellar density within the GCs. In this section we will present the details of the matching procedure.

To match the photometric data for each of the clusters we use the ACS images, the Stetson source catalogs with R.A. and decl. coordinates and the $\operatorname{IRAF}^{17}$ task tfinder. The latter is used to adjust the plate solution of the ACS images to correspond with the R.A. and decl. coordinates given by Stetson. The required input information includes: the pixel scale of the ACS images (0'.05), the observation equinox (J2000.0), and the world coordinates and pixel coordinates of the reference pixel within the ACS image.

The new plate solution is then used in IRAF/wcsctran to convert the R.A. and decl. values of the Stetson catalogs into corresponding $x y$-pixel coordinates in the ACS images. This requires that a number $(\gg 3)$ of stars in the Stetson catalog are unmistakably identified in the ACS images, and are widely distributed over the FOV. Table 1 gives the number of stars in each cluster used to calculate the new plate solution (Column 2), as well as how much the original R.A. and decl. for each star deviates from the one derived from the new plate solution and the $x y$-pixel position. There are no multiple matches in any of the merged catalogs, only the best fitting pair of ACS and Stetson detections are included in the data base.

Although the ACS and Stetson GC samples have 43 objects in common only 34 were suitable for our study. The remaining 9 GCs (NGC 5024, NGC 5986, NGC 6101, NGC 6121, NGC 6218, NGC 6254, NGC 6584, NGC 6656 and NGC 6723) do not overlap sufficiently in their FOV to re-calculate the plate solution and hence to derive their $x y$-pixel position with the required accuracy. We note that in the final catalog, containing both ACS and Stetson photometry, the R.A. and decl. coordinates are based on the high quality ACS astrometry. The Stetson world-coordinates are used as a starting point to obtain ACS pixel coordinates only.

To match the ACS and Stetson photometry we use TOPCAT (e.g., Taylor 2005) and compare the $x y$-pixel coordinates of each detected star using the match option "two-dimensional (2D) Cartesian." We consider a star to match between both catalogs if the separation between the ACS and Stetson coordinates is not greater than 1.5 ACS pixel, i.e., 0'.075. The limit is based on the accuracy of the R.A. and decl. coordinates (see Table 1, Columns 3 and 4) obtained with tfinder, which is less than 0.'05 in R.A. and decl., corresponding to approximately 1 ACS pixel in each dimension. The 2D-pixel positions of matched objects should therefore differ by no more than 1.5 ACS pixels. Limiting the maximum pixel offset in such a way will inevitably reduce the number of matched stars in the combined ACS-Stetson catalog, but it will also reduce the probability of mismatches in the more crowded, central regions of an individual cluster.

For each cluster in our sample we derived the number of stars with one or more neighbors within the matching radius (as applied in the catalog matching), which we consider bonafide candidates for mismatches. The ratio between those stars and the total number of stars in the original ACS catalog is a measure for the possible false matches. Obviously, this mismatch rate depends on the position of a star, or its local stellar density.

\footnotetext{
17 IRAF is distributed by the National Optical Astronomy Observatories, which are operated by the Association of Universities for Research in Astronomy, Inc., under cooperative agreement with the National Science Foundation.
} 
Table 1

Results of the Catalog Matching Procedure

\begin{tabular}{|c|c|c|c|c|}
\hline Cluster & NumPS $^{a}$ & $\begin{array}{l}\operatorname{rms}_{\mathrm{R} . A .} \\
(\operatorname{arcsec})\end{array}$ & $\begin{array}{l}\mathrm{rm}_{\mathrm{decl}} . \\
(\operatorname{arcsec})\end{array}$ & Num $_{\text {match }}{ }^{b}$ \\
\hline Е 0003 & 50 & 0.0215775 & 0.0205797 & 207 \\
\hline NGC 0104 & 12 & 0.0094098 & 0.0157275 & 36 \\
\hline NGC 0288 & 19 & 0.0106115 & 0.0111964 & 108 \\
\hline NGC 0362 & 23 & 0.0515514 & 0.0383049 & 118 \\
\hline NGC 1261 & 14 & 0.0049360 & 0.0084422 & 49 \\
\hline NGC 1851 & 19 & 0.0216507 & 0.0147235 & 54 \\
\hline NGC 2298 & 17 & 0.0079661 & 0.0105671 & 86 \\
\hline NGC 2808 & 15 & 0.0133545 & 0.0175066 & 148 \\
\hline NGC 3201 & 18 & 0.0129494 & 0.0053085 & 66 \\
\hline NGC 4147 & 38 & 0.0409929 & 0.0286021 & 255 \\
\hline NGC 4590 & 23 & 0.0141201 & 0.0055300 & 44 \\
\hline NGC 4833 & 24 & 0.0281217 & 0.0208677 & 35 \\
\hline NGC 5053 & 23 & 0.0163323 & 0.0304663 & 35 \\
\hline NGC 5139 & 19 & 0.0198540 & 0.0154449 & 49 \\
\hline NGC 5272 & 27 & 0.0197264 & 0.0111676 & 46 \\
\hline NGC 5286 & 20 & 0.0294617 & 0.0171257 & 26 \\
\hline NGC 5466 & 12 & 0.0688927 & 0.0221558 & 9 \\
\hline NGC 5904 & 46 & 0.0165915 & 0.0132720 & 260 \\
\hline NGC 5927 & 19 & 0.0170713 & 0.0138404 & 14 \\
\hline NGC 6093 & 6 & 0.0311338 & 0.0022479 & 6 \\
\hline NGC 6171 & 7 & 0.0111846 & 0.0030558 & 8 \\
\hline NGC 6205 & 21 & 0.0168024 & 0.0200496 & 105 \\
\hline NGC 6341 & 40 & 0.0235042 & 0.0145158 & 631 \\
\hline NGC 6352 & 21 & 0.0183443 & 0.0204583 & 75 \\
\hline NGC 6362 & 13 & 0.0182156 & 0.0153341 & 18 \\
\hline NGC 6397 & 25 & 0.0243064 & 0.0083323 & 112 \\
\hline NGC 6441 & 23 & 0.0255059 & 0.0174784 & 83 \\
\hline NGC 6541 & 30 & 0.0335179 & 0.0484386 & 77 \\
\hline NGC 6752 & 21 & 0.0178754 & 0.0121514 & 46 \\
\hline NGC 6809 & 41 & 0.0297873 & 0.0173643 & 149 \\
\hline NGC 6838 & 19 & 0.0247012 & 0.0084462 & 25 \\
\hline NGC 7078 & 44 & 0.0373419 & 0.0167781 & 239 \\
\hline NGC 7089 & 32 & 0.0404868 & 0.0290307 & 83 \\
\hline NGC 7099 & 35 & 0.0279548 & 0.0169936 & 50 \\
\hline
\end{tabular}

Notes.

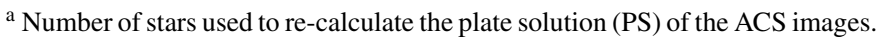

${ }^{b}$ Number of stars with matched ACS and Stetson photometry, i.e., offset position $\leqslant 1.5$ pixel.

Given that the corresponding Stetson stars, due to the lower spatial resolution of the data, are mostly found in the outer regions of the ACS FOV we can assume that the number of mismatches in the combined ACS-Stetson catalogs is much lower. We find that no cluster has more than $4 \%$ stars with close neighbors. This ratio is higher for NGC 6441 (4.36\%), but as we will describe in Section 3.1, this cluster will be excluded from further analysis. Including the high accuracy of the transformed pixel coordinates (see Table 1) we assume that the closet match, as found by TOPCAT, has a low risk of being a mismatch.

The photometric offset, as used in the further analysis, is always calculated as the difference between the transformed ACS magnitudes and its Stetson counterpart: $V_{\mathrm{ACS}}-V_{\text {Stetson }}$ and $I_{\mathrm{ACS}}-I_{\text {Stetson }}$, respectively.

\section{PHOTOMETRIC OFFSETS RELATIVE TO CLUSTER PROPERTIES}

Following the matching of the photometric catalogs, we apply one additional selection criterion to the stellar sample of each GC. Based on the ACS photometry, we reject all stars that were saturated on the ACS images. Even though (Gilliland 2004) has shown that $1 \%$ photometry can be achieved for saturated stars on the ACS chips, we have decided to be conservative and eliminate these stars from the comparisons presented in this paper. As shown in Table 2, this reduces the total number of stars in NGC 6093 from 6 to only 2. Therefore, we will exclude this cluster from later analyses due to its low number statistics. For comparison we also derived the photometric offset between the two data sets, as well as the correlation coefficients for the complete matched catalogs, i.e., without applying any selection criteria. Although the mean offset for both filters is smaller, its standard deviation increases. Without going more into detail we find all correlations studied here to be weaker than for the selected sample, and will focus on the selected sample for the remainder of this study.

\subsection{Observational Properties: Magnitude, Color, and Photometric Errors}

A comparison between the GC color-magnitude diagrams (CMDs) for selected globular clusters ${ }^{18}$ is shown in Figure 1, where black symbols represent the transformed ACS $V$ - and $I$-band data, while red symbols correspond to their Stetson counterparts. In general we find that some clusters show very good agreement between the space-based and ground-based photometry (e.g., NGC 4147, NGC 5904, NGC 6397), as well as some for which either the $V$ - or I-band photometry show a significant offset (e.g., E 3), reaching up to $\sim 0.3 \mathrm{mag}$. The specific offsets become easier to follow when we plot star-by-star differences between the transformed and observed magnitudes in both filters, shown for the selected clusters in Figures 2 and 3. For example, in the case of E3, the matched photometric catalog contains 207 stars following the selection criteria as described in Section 2.

The difference between the transformed ACS $V$-band and the Stetson photometry shows a clear negative trend with $(V-I)$ color, whereas no such trend is seen in the I-band diagram. On the other hand, NGC 6441 features a negative slope in the $\Delta V$ versus $(V-I)$ relation and a positive one in $\Delta I$ versus $(V-I)$. The transformed $V$-band photometry is also brighter than its Stetson counterpart, with the difference increasing as color increases. In contrast, the ACS I-band is fainter, the effect being stronger for redder stars. This trend differs from that of all of the other clusters, for which the ACS I-band follows the trend of the $V$-band being the brighter one. At this point, we are unable to trace the reason for this unusual behavior, but note that the original source catalog by Stetson is based on the fewest number of observations. We therefore exclude NGC 6441 from the correlation analysis.

We combine the data for all of the clusters and compare the transformed ACS magnitudes with their Stetson counterparts. Figure 4 shows the complete sample with the transformed ACS $(V-I)$ color and the photometric offset in both filters. For both filters we find a population of stars that do not follow the general trend, i.e., showing a larger photometric offset than the bulk of the stars with the same color. These stars are plotted as open diamonds and belong almost exclusively to $\mathrm{E} 3$. The resulting correlation coefficients between the offset and the $(V-I)$ color (as given by Stetson) of the individual stars (excluding E 3), are 0.02 for the $\Delta V$ versus $(V-I)_{\text {Stetson }}$ relation and -0.06 for the $\Delta I$ versus $(V-I)_{\text {Stetson }}$ relation.

In general, the transformed $I$-band magnitudes are brighter than their Stetson counterparts, and show a smaller spread

\footnotetext{
18 All plots and matched ACS-Stetson catalogs are available upon request.
} 
Table 2

Mean and Median Offset between the Converted ACS V-and I-band Photometry and the Stetson Standard Field Catalogs

\begin{tabular}{|c|c|c|c|c|c|c|c|c|c|c|}
\hline Number & $\Delta V_{\text {mean }}$ & $\sigma_{V}$ & $\Delta I_{\text {mean }}$ & $\sigma_{I}$ & $N_{\text {match }}$ & {$[\mathrm{M} / \mathrm{H}]_{\mathrm{GC}}{ }^{\mathrm{a}}$} & $\mathrm{Age}^{\mathrm{b}}$ & $\Delta \mathrm{Age}^{\mathrm{b}}$ & $\begin{array}{c}l^{\mathrm{c}} \\
(\mathrm{deg})\end{array}$ & $\begin{array}{c}b^{\mathrm{c}} \\
(\mathrm{deg})\end{array}$ \\
\hline Е 0003 & -0.1345 & 0.0293 & -0.0990 & 0.0254 & $207^{d}$ & -0.69 & 1.02 & 0.15 & 292.27 & -19.02 \\
\hline NGC 0104 & -0.0252 & 0.1062 & -0.0548 & 0.1002 & 19 & -0.64 & 1.05 & 0.09 & 305.90 & -44.89 \\
\hline NGC 0288 & -0.0452 & 0.0477 & -0.0687 & 0.0517 & 100 & -0.92 & 0.83 & 0.03 & 152.28 & -89.38 \\
\hline NGC 0362 & -0.0610 & 0.1633 & -0.0634 & 0.1724 & 100 & -0.87 & 0.81 & 0.05 & 301.53 & -46.25 \\
\hline NGC 1261 & -0.0290 & 0.1120 & -0.0462 & 0.0633 & 38 & -0.86 & 0.79 & 0.05 & 270.54 & -52.13 \\
\hline NGC 1851 & -0.0662 & 0.0667 & -0.0925 & 0.0861 & 52 & -0.81 & 0.75 & 0.04 & 244.51 & -35.04 \\
\hline NGC 2298 & -0.0351 & 0.0607 & -0.0498 & 0.0473 & 71 & -1.49 & 0.99 & 0.05 & 245.63 & -16.01 \\
\hline NGC 2808 & -0.0641 & 0.0865 & -0.0894 & 0.0837 & 108 & -0.89 & 0.85 & 0.02 & 282.19 & -11.25 \\
\hline NGC 3201 & -0.0225 & 0.0640 & -0.0521 & 0.0311 & 36 & -1.02 & 0.81 & 0.03 & 277.23 & 8.64 \\
\hline NGC 4147 & -0.0278 & 0.0412 & -0.0272 & 0.0371 & 232 & -1.28 & 0.89 & 0.03 & 252.85 & 77.19 \\
\hline NGC 4590 & -0.0197 & 0.0304 & -0.0413 & 0.0199 & 26 & -1.78 & 0.91 & 0.04 & 299.63 & 36.05 \\
\hline NGC 4833 & -0.0568 & 0.0722 & -0.0815 & 0.0701 & 33 & -1.49 & 0.98 & 0.05 & 303.61 & -8.01 \\
\hline NGC 5053 & -0.0285 & 0.0615 & -0.0477 & 0.0203 & 27 & -1.76 & 0.96 & 0.04 & 335.69 & 78.94 \\
\hline NGC 5139 & -0.0321 & 0.0941 & -0.0721 & 0.1137 & 19 & -1.13 & 0.89 & 0.05 & 309.10 & 14.97 \\
\hline NGC 5272 & -0.0438 & 0.0427 & -0.0586 & 0.0451 & 27 & -1.12 & 0.89 & 0.04 & 42.21 & 78.71 \\
\hline NGC 5286 & -0.0183 & 0.0598 & -0.0506 & 0.0469 & 21 & -1.19 & 0.98 & 0.05 & 311.61 & 10.57 \\
\hline NGC 5466 & -0.0450 & 0.0263 & -0.0460 & 0.0301 & 7 & -1.98 & 1.07 & 0.05 & 42.15 & 73.59 \\
\hline NGC 5904 & -0.0727 & 0.0825 & -0.0815 & 0.0655 & 208 & -0.90 & 0.83 & 0.02 & 3.86 & 46.80 \\
\hline NGC 5927 & -0.0584 & 0.0689 & -0.0547 & 0.1161 & 9 & -0.50 & 1.01 & 0.11 & 326.60 & 4.86 \\
\hline NGC 6093 & 0.0650 & 0.1273 & 0.0140 & 0.1061 & 2 & -1.25 & 0.98 & 0.05 & 352.67 & 19.46 \\
\hline NGC 6171 & -0.0364 & 0.0346 & -0.0397 & 0.0235 & 8 & -0.81 & 1.13 & 0.08 & 3.37 & 23.01 \\
\hline NGC 6205 & -0.0152 & 0.0665 & -0.0411 & 0.0727 & 51 & -1.11 & 0.90 & 0.04 & 59.01 & 40.91 \\
\hline NGC 6341 & -0.0191 & 0.0446 & -0.0342 & 0.0438 & 564 & -1.94 & 1.03 & 0.05 & 68.34 & 34.86 \\
\hline NGC 6352 & -0.0434 & 0.0394 & -0.0582 & 0.0340 & 61 & -0.56 & 1.02 & 0.10 & 341.42 & -7.17 \\
\hline NGC 6362 & -0.0028 & 0.0276 & 0.0080 & 0.0257 & 14 & -0.85 & 1.07 & 0.06 & 325.55 & -17.57 \\
\hline NGC 6397 & -0.0395 & 0.0244 & -0.0373 & 0.0248 & 112 & -1.54 & 0.99 & 0.04 & 338.17 & -11.96 \\
\hline NGC 6441 & -0.0803 & 0.0631 & 0.1311 & 0.0842 & 49 & -0.46 & 0.85 & 0.09 & 353.53 & -5.01 \\
\hline NGC 6541 & -0.0678 & 0.0671 & -0.0791 & 0.0670 & 77 & -1.31 & 1.01 & 0.04 & 349.48 & -11.09 \\
\hline NGC 6752 & -0.0460 & 0.0452 & -0.0451 & 0.0466 & 36 & -1.02 & 0.92 & 0.04 & 336.49 & -25.63 \\
\hline NGC 6809 & -0.0600 & 0.0525 & -0.0861 & 0.0619 & 149 & -1.32 & 0.96 & 0.05 & 8.80 & -23.27 \\
\hline NGC 6838 & -0.0254 & 0.0298 & -0.0275 & 0.0390 & 22 & -0.59 & 1.11 & 0.10 & 56.74 & -4.56 \\
\hline NGC 7078 & -0.0050 & 0.0891 & -0.0159 & 0.1108 & 123 & -1.80 & 1.01 & 0.04 & 65.01 & -27.31 \\
\hline NGC 7089 & -0.0424 & 0.0769 & -0.0671 & 0.0767 & 60 & -1.09 & 0.91 & 0.05 & 53.38 & -35.78 \\
\hline NGC 7099 & -0.0415 & 0.0339 & -0.0477 & 0.0301 & 49 & -1.70 & 1.01 & 0.04 & 27.18 & -46.83 \\
\hline Average & -0.0396 & 0.0629 & -0.0471 & 0.0610 & & & & & & \\
\hline
\end{tabular}

Notes. Boldface values represent the average offset and average standard deviation for all 34 GCs.

${ }^{\text {a }}$ From Carretta \& Gratton (1997).

b From Marín-Franch et al. (2009).

c From Harris (1996).

${ }^{\mathrm{d}}$ One star was removed from the sample, its offset $>0.5 \mathrm{mag}$, positioned at the edge of the ACS field of view.

around the "zero" line as compared with the $V$-band. The mean offset for the complete stellar sample (in total 2461 stars) and their standard deviations (again excluding $\mathrm{E} 3$ ) are calculated as $(\overline{\Delta V}=-0.0382 ; \sigma=0.0703)$ and $(\overline{\Delta I}=-0.0519 ; \sigma=$ 0.0711 ), which is in agreement with the result based on the average offset for 34 MWGCs.

Before we discuss the correlation between the photometric offsets and GC properties, we need to consider the possibility that differences in the photometry are due to the magnitude of the stars and/or the photometric accuracy. Figures 5 and 6 show, for each cluster separately, the photometric offset in both filters as a function of the ACS magnitudes and of the corresponding photometric errors. As in the $(V-I)$ color plots, we find a wide range of features. Some, e.g., NGC 0104, NGC 2808 show the largest offset for the brighter, although not saturated stars, and despite the fact that they have the smallest photometric errors. In contrast, we find that the brighter stars in NGC 5904 and NGC 6809 show smaller photometric offsets as compared with the fainter ones. However, in general there is no significant systematic correlation between the photometric errors and the photometric offsets.

\subsection{Integrated and Resolved Color, Metallicity, and Mass Composite Populations}

Our sample of MWGCs allows us to search for correlations between the photometric offsets and GC properties such as metallicity, integrated color, HB morphology, and GC mass. For our analysis, we use the mean difference between the transformed ACS magnitude and its counterpart as given by Stetson (2000, 2005). In addition, we also investigate the correlations between different GC parameters and the spread of the offset, i.e., the standard deviation, in particular to assess the significance of the correlation between the offset and GC parameters. In all of the following figures, open symbols represent the (mean) photometric offset, whereas filled symbols represent the standard deviation $\sigma$. A large open triangle marks NGC 2419 , whose photometry was originally used to 


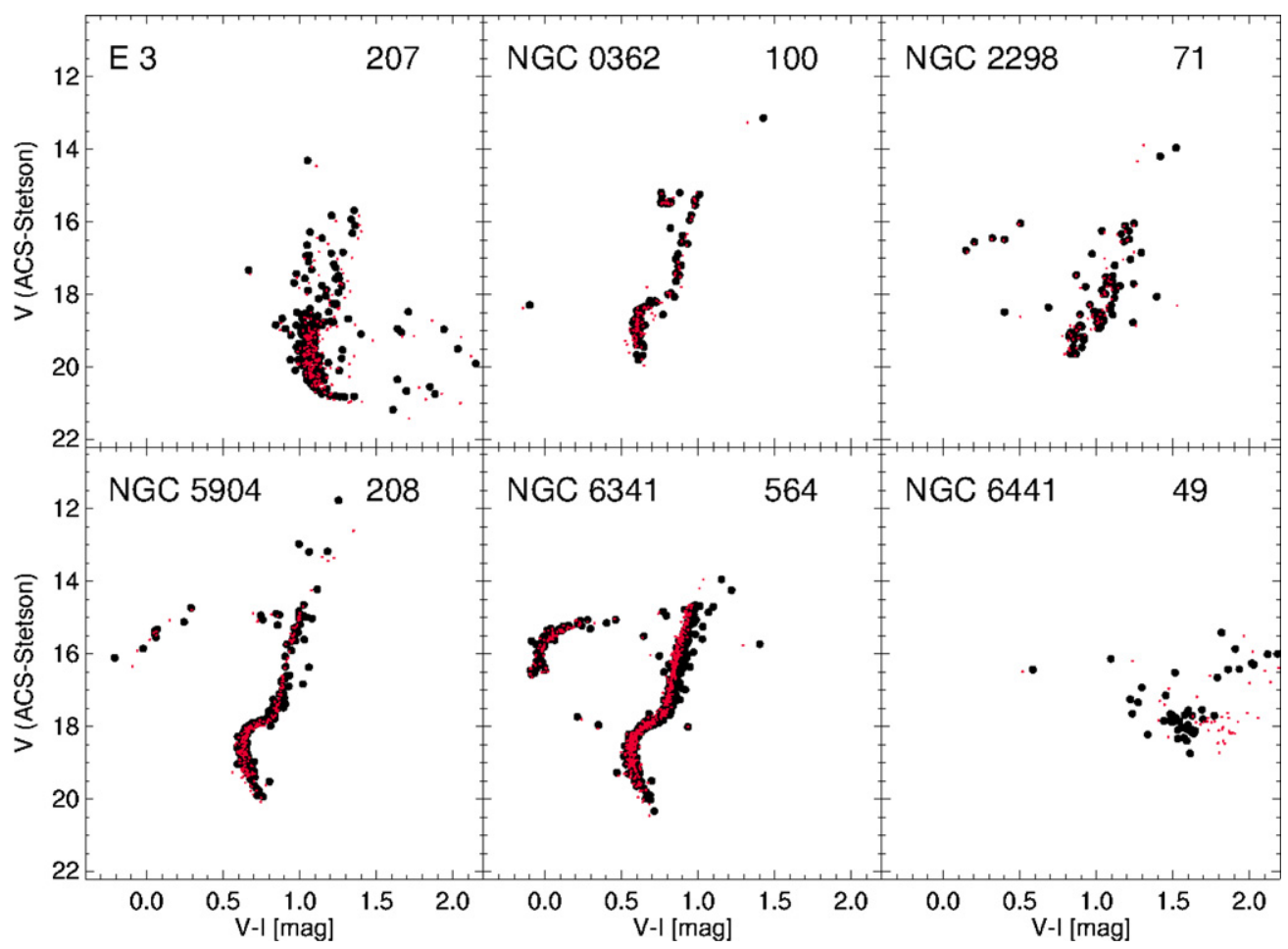

Figure 1. $V$ vs. $(V-I)$ color-magnitude diagrams of six selected MWGCs from our sample, based on the transformed ACS $V$-and $I$-band magnitudes (black symbols) and their Stetson counterparts (red symbols).

(A color version of this figure is available in the online journal.)

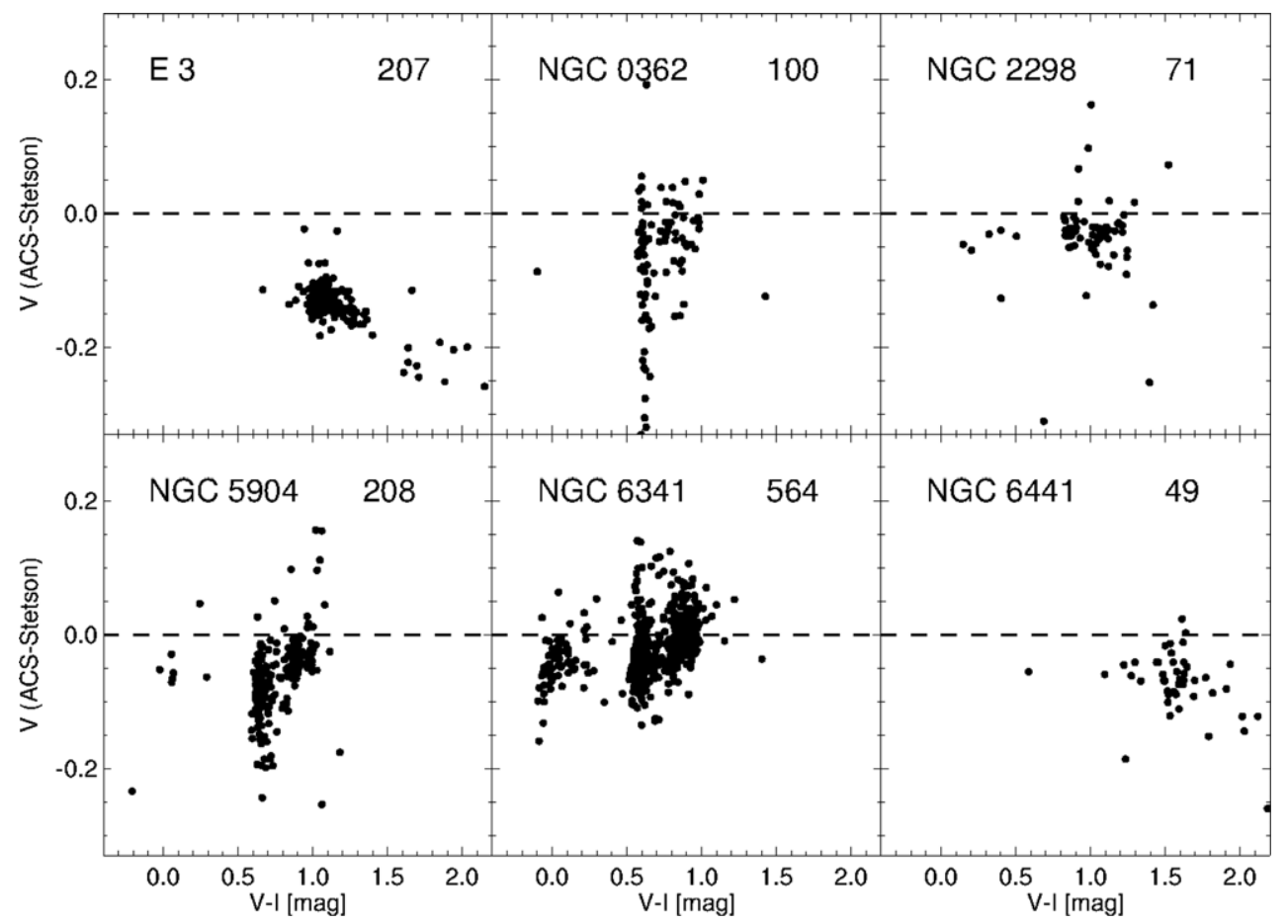

Figure 2. $V$-band magnitude difference (ACS-Stetson) for six MWGCs as a function of the transformed $(V-I)$ color index. The number of stars found in both data sets is given in the upper right corner of each panel. The horizontal (dashed) line marks zero offset.

test the transformation equations (Sirianni et al. 2005). In the discussion of our results we will also include $\operatorname{Prob}_{N}\left(|r| \geqslant\left|r_{0}\right|\right)$, the probability (non-directional) that the Pearson correlation coefficient $|r|$ between the photometric offset and a given cluster parameter, could also be based on an uncorrelated sample. Hence, the higher the probability, which we will call the significance level, the less significant is the correlation. We note that $N$ is the number of data points $(\gtrsim 31)$, and depends on the cluster parameter, e.g., E 3 does NOT have an integrated $(V-I)$ value. All of the correlations and related quantities for the $V$-band are listed in Table 3 and those for the $I$-band are in Table 4. 


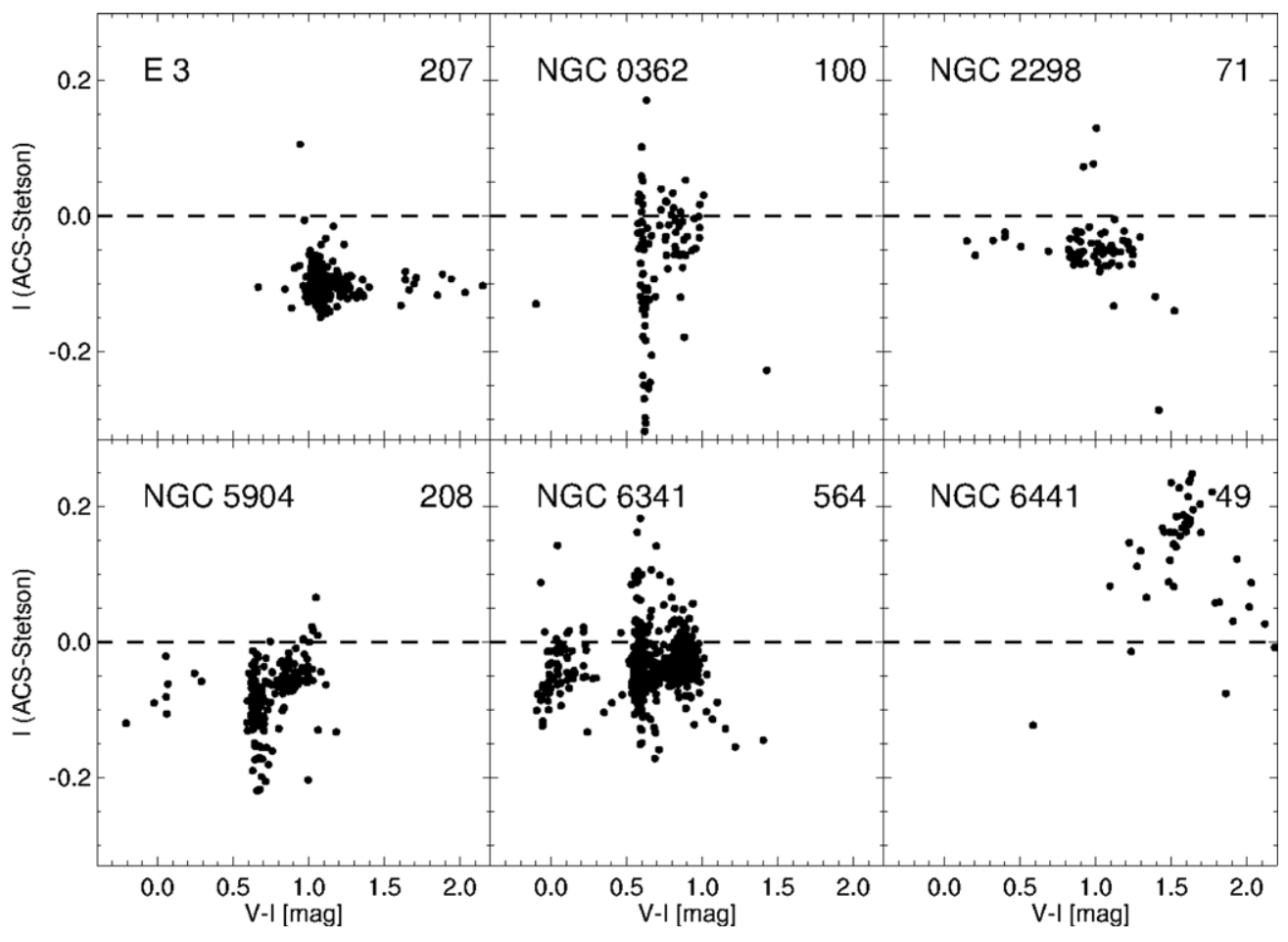

Figure 3. Same as Figure 2, but for the $I$-band magnitude difference.
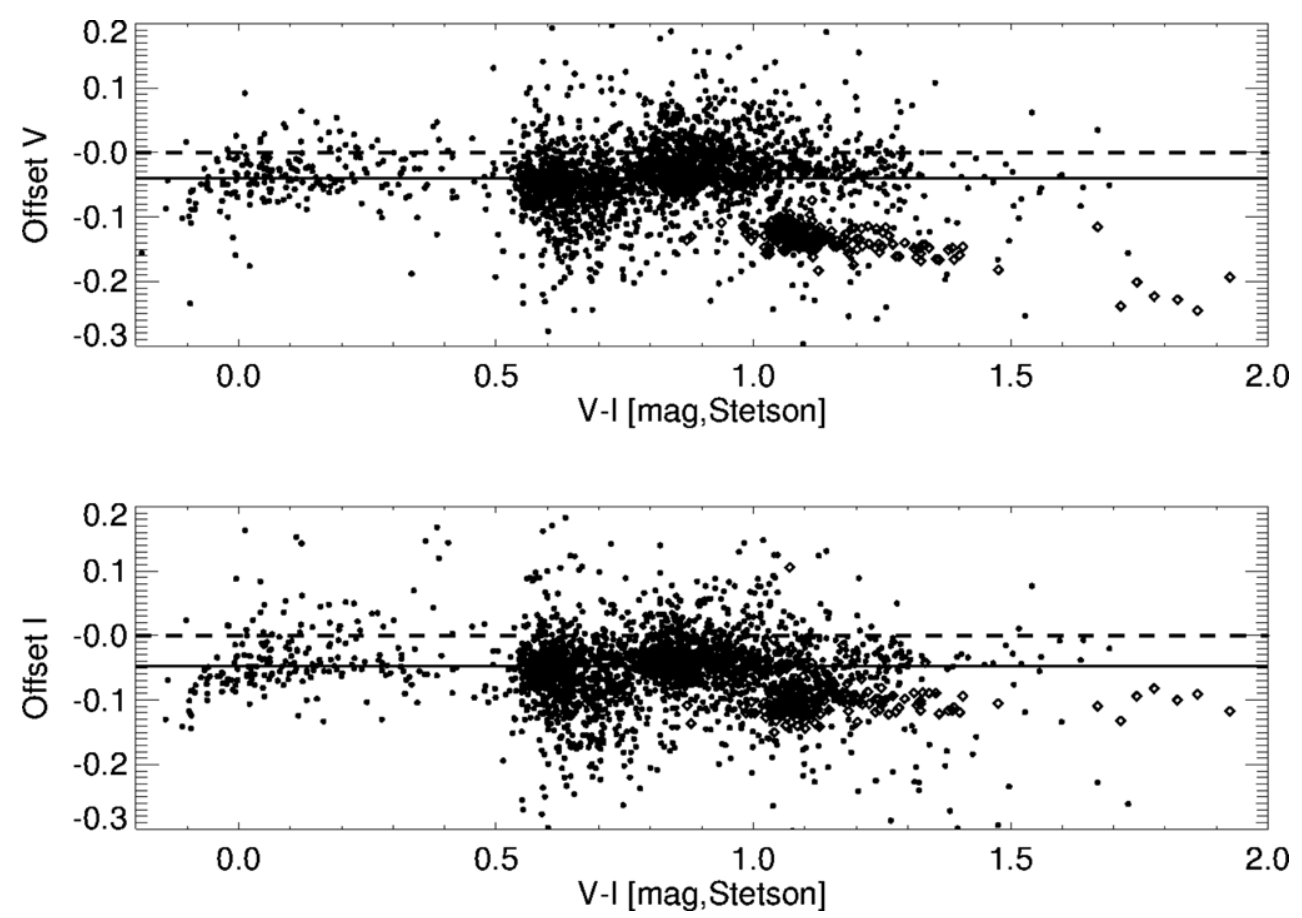

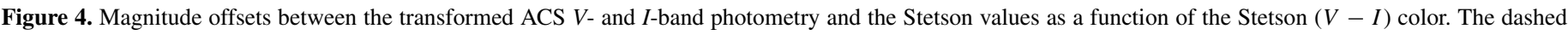
line marks zero offset, whereas the solid line represents the mean offset derived as the average offset for 34 MWGCs (see Table 2).

\subsubsection{Integrated/Resolved $(V-I)$ Color}

In Figure 7 we show the $\Delta V$ and $\Delta I$ offsets as a function of the integrated $(V-I)$ color of each cluster and the corresponding correlation coefficients. We are interested in this correlation since the integrated color is based only on observations and does not include any stellar population models, but also because it depends on the cluster metallicity. We would therefore expect the correlations between photometric offset and either integrated color and metallicity to be conform. The $(V-I)$ colors were corrected for galactic extinction, where both $(V-I)_{\mathrm{GC}}$ and $E(B-V)_{\mathrm{GC}}$ were taken from (Harris 1996, 2010 online edition). The latter was converted into $E(V-I)$ using the prescription by Cardelli et al. (1989) and Barmby et al. (2000): $E(V-I)=$ $1.26 * E(B-V)$.

The linear correlation coefficient between the photometric offset and the integrated $(V-I)$ color, as well as the corresponding significance levels are given in Tables 3 and 4 .

There is no significant correlation between the integrated color and the photometric offset in our sample, the same 


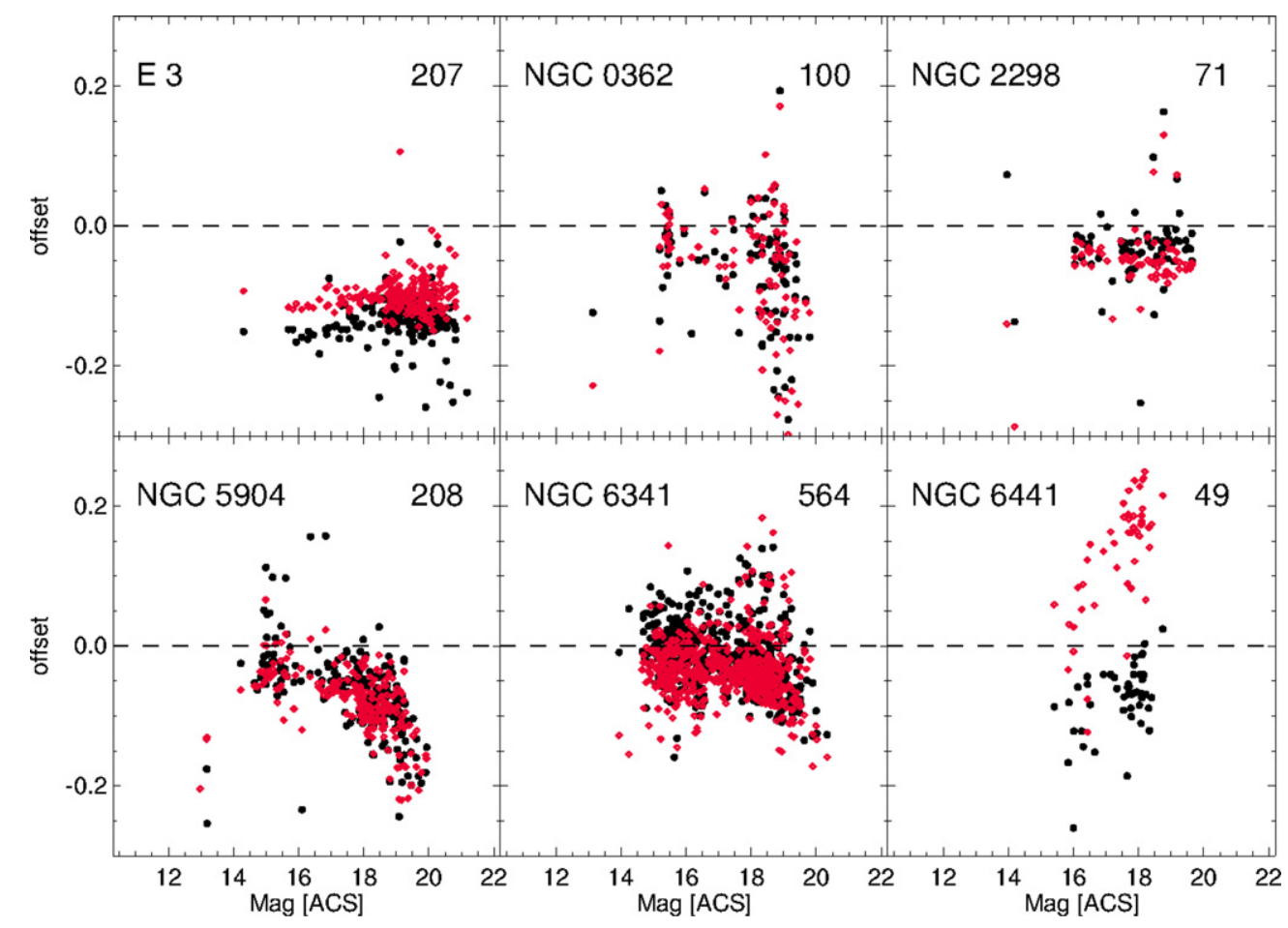

Figure 5. Photometric offset between the transformed ACS $V$ - and $I$-band and the Stetson values as a function of the ACS luminosity. Filled (black) dots refer to the $V$-band, whereas open (red) symbols correspond to the $I$-band. The horizontal (dashed) line marks zero offset.

(A color version of this figure is available in the online journal.)

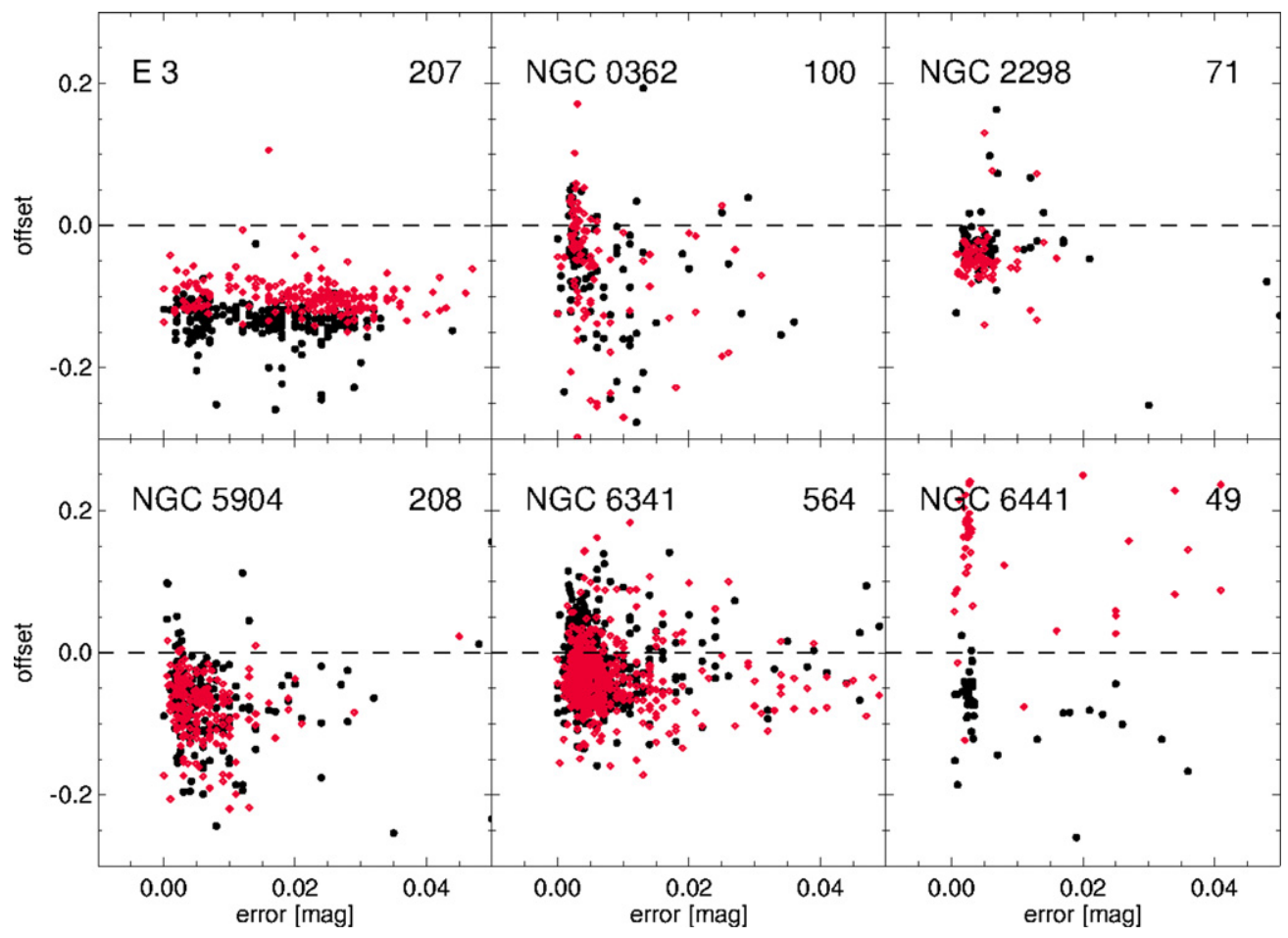

Figure 6. Photometric offset in the $V$ - and $I$-band as a function of the ACS photometric error. The symbols are as in Figure 5. The horizontal (dashed) line marks zero offset.

(A color version of this figure is available in the online journal.)

is valid for uncorrected $(V-I)$ colors. However, based on the offset in the $I$ band (see Figure 7 , right panel) clusters bluer than NGC 2419 show a much stronger correlation compared to the complete $V-I$ color range. Selecting only clusters with $(V-I) \leqslant(V-I)_{\mathrm{NGC} 2419}$, the correlation coefficients are corr $=-0.3954$; Prob $=0.0277$ for the $V$ band and corr $=-0.6135$; Prob $=0.0002$ for the $I$ band. 

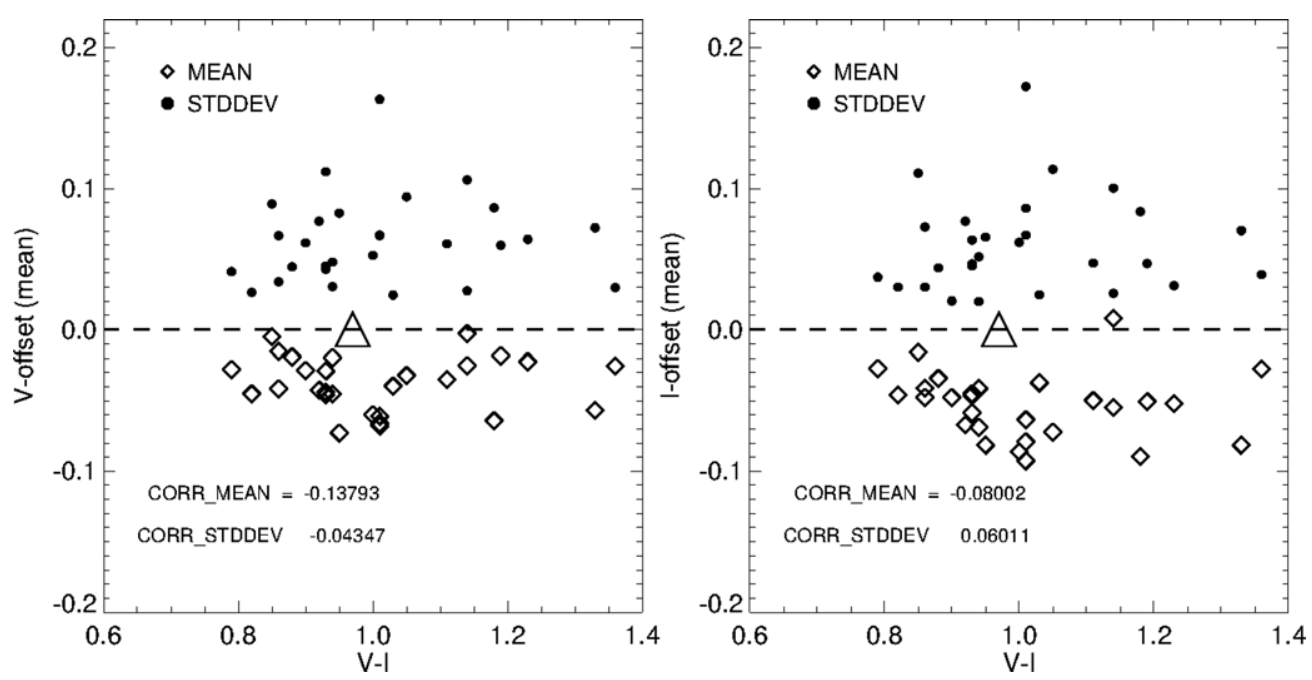

Figure 7. Photometric offset in the $V$ - and $I$-band (offset = ACS-Stetson) as a function of the globular cluster $(V-I)$ color index. The dashed line represents "zero" offset and the open triangle represents NGC 2419. As before the $(V-I)$ colors are corrected for reddening.

Table 3

Linear Correlation Coefficients between Various Cluster Parameters and the Photometric Offset (Mean), and Its Standard Deviation in the $V$ Band

\begin{tabular}{lllr}
\hline \hline Parameter & $\Delta V_{\text {mean }}$ & Prob & stddev $_{V}$ \\
\hline$(V-I)$ & -0.119 & 0.255 & 0.0284 \\
{$[\mathrm{M} / \mathrm{H}]$} & -0.3038 & 0.091 & 0.2243 \\
$\mathrm{HBR}$ & -0.0234 & 0.451 & -0.2767 \\
$\Delta(V-I)$ & -0.0668 & 0.363 & -0.1949 \\
Mass & -0.0229 & 0.903 & 0.4700
\end{tabular}

Note. NGC 6441 and NGC 6093 are excluded due to the low number of stars.

Table 4

Linear Correlation Coefficients between Various Cluster Parameters, the Mean Photometric Offset, and Its Standard Deviation in the $I$ Band

\begin{tabular}{lccr}
\hline \hline Parameter & $\Delta I_{\text {mean }}$ & Prob & \multicolumn{1}{c}{ stddev $_{I}$} \\
\hline$(V-I)$ & -0.0905 & 0.299 & -0.0543 \\
{$[\mathrm{M} / \mathrm{H}]$} & -0.2391 & 0.187 & 0.2651 \\
$\mathrm{HBR}$ & -0.0769 & 0.343 & -0.2748 \\
$\Delta(V-I)$ & -0.1748 & 0.178 & -0.2024 \\
Mass & -0.3089 & 0.091 & 0.5351 \\
\hline
\end{tabular}

Note. NGC 6441 and NGC 6093 are excluded.

In comparison, there is no significant correlation between magnitude offset and integrated $(V-I)$ cluster color for objects redder then NGC 2419.

Since the integrated GC color depends on the underlying stellar populations, and also on a proper correction for galactic extinction, the weak correlations between integrated color and photometric offset become understandable. In contrast, when we compare the correlation coefficients for both the $V$ - and $I$-band filters within an individual cluster, as shown in Figure 8, we find them to be consistent. That is to say, a correlation between $V$-band offset and resolved individual (stellar) $(V-I)$ color is mirrored by a correlation between $I$-band offset and color. The correlation between the two filters was derived to be 0.6138 , with a high level of significance $\left(\operatorname{Prob}_{N}\left(|r| \geqslant\left|r_{0}\right|\right)=\right.$ 0.00015).

In Figure 9 we select only the HB stars, because they cover a wide range of $(V-I)$ color, and also belong to the same

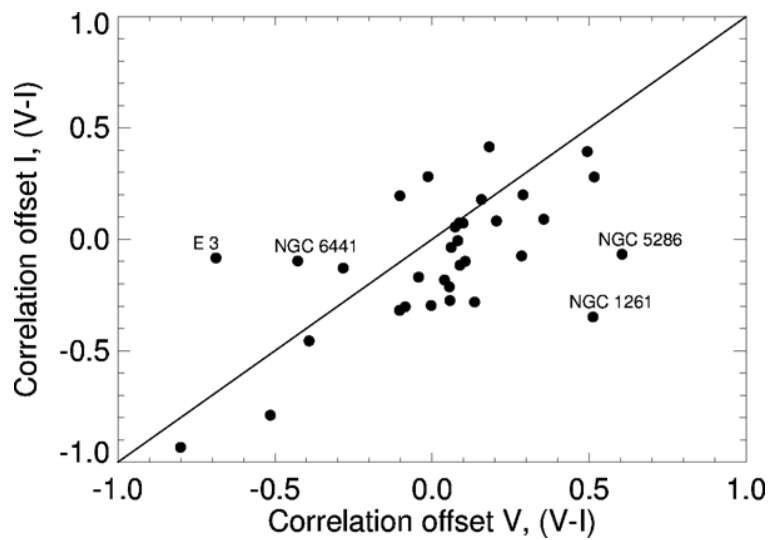

Figure 8. Correlation coefficient between $V$ - and $I$-band offset and the resolved $(V-I)$ color. For E3, NGC 6441, NGC 1261 and NGC 5286 the correlation between the integrated $V-I$ color and the photometric offset in $V$ is not mirrored by an equally strong correlation between $V-I$ and the offset in the $I$-band. Not shown is NGC 6093, due to the low number of contributing stars (2).

evolutionary phase. We select only clusters with a well defined HB, e.g., NGC 0288, NGC 0362, NGC 1851, NGC 2808, NGC 3201, NGC 4147, NGC 5904, NGC 6341, and NGC 7078. We find that the correlation between the photometric offset and the $(V-I)$ color of the individual stars varies significantly. Interestingly, the strongest correlation between photometric offset and $(V-I)$ is found in clusters with the most evenly populated HBs, i.e., HBR $\sim 0$. For example, for NGC 5904 $(\mathrm{HBR}=0.31)$ and NGC $3201(\mathrm{HBR}=0.05)$, the correlation coefficients were estimated to be $\left[\operatorname{corr}_{V}=0.673, \operatorname{corr}_{I}=\right.$ 0.741], and [ $\left.\operatorname{corr}_{V}=0.599, \operatorname{corr}_{I}=0.692\right]$, respectively. In contrast, clusters with a very blue or very red HB, e.g., NGC 0288, or NGC 0362, show very little correlation between the photometric offset and the $(V-I)$ color. We note that these are also the GCs for which the HB structure implies a second parameter spread, given their very different HBs despite their similar metallicities (see Section 3.2.2.)

The HB morphology is one of the most discerning GC parameters, i.e., revealing differences in the chemical composition beyond the metallicity, e.g., in NGC 0288 and NGC 0362 (see above). Therefore, we also examined the correlation between the photometric offset for each cluster and the 


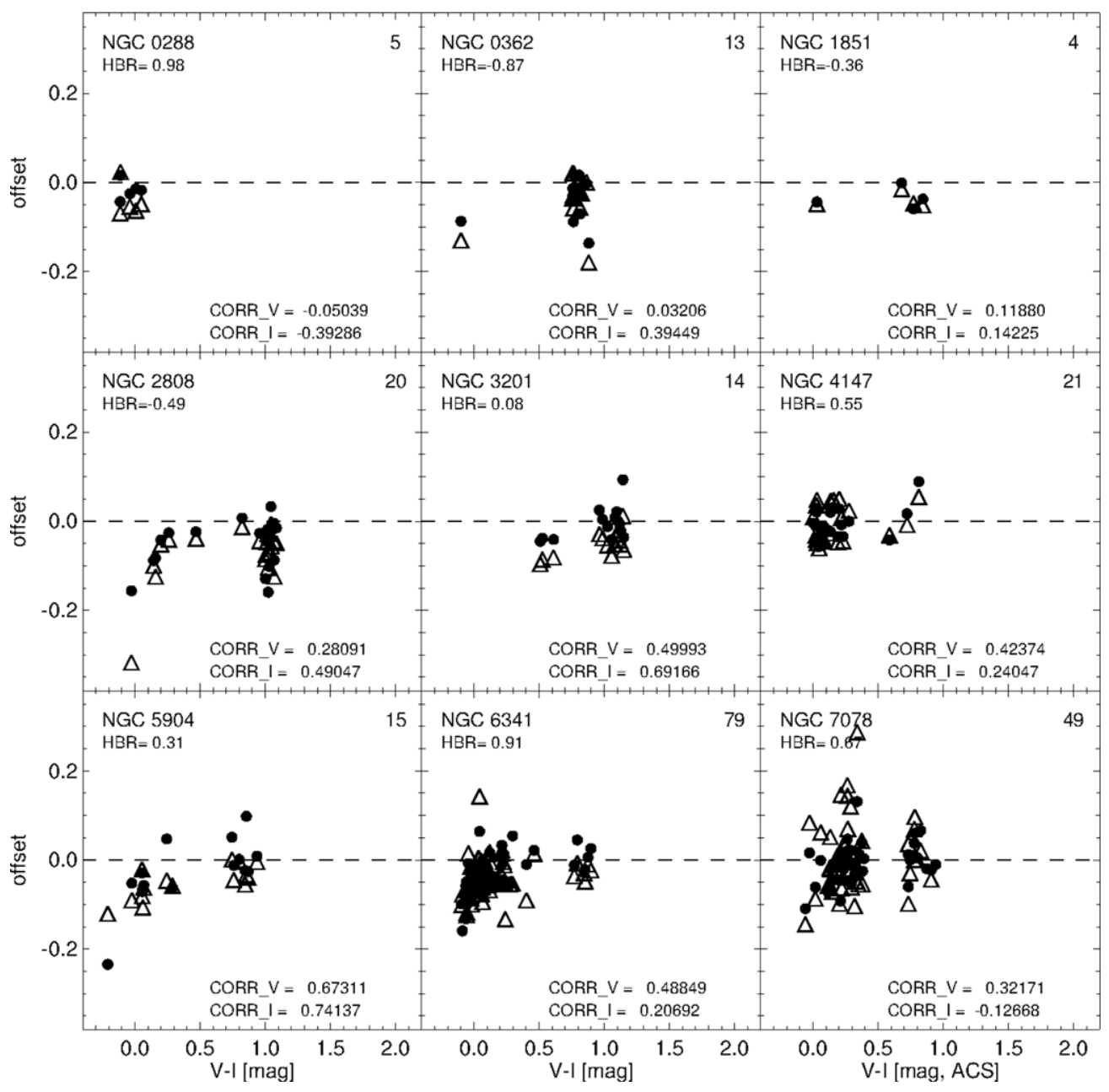

Figure 9. Photometric offset in the $V$ and $I$ bands for globular clusters with a distinct HB. Filled circles refer to the $V$ band, whereas open triangles represent the I-band data. At the top left we give the name of the cluster and its HBR (Harris 1996). The number of HB stars is given at the right. Each panel also gives the linear correlation coefficient between $V-I$ color and photometric offset.
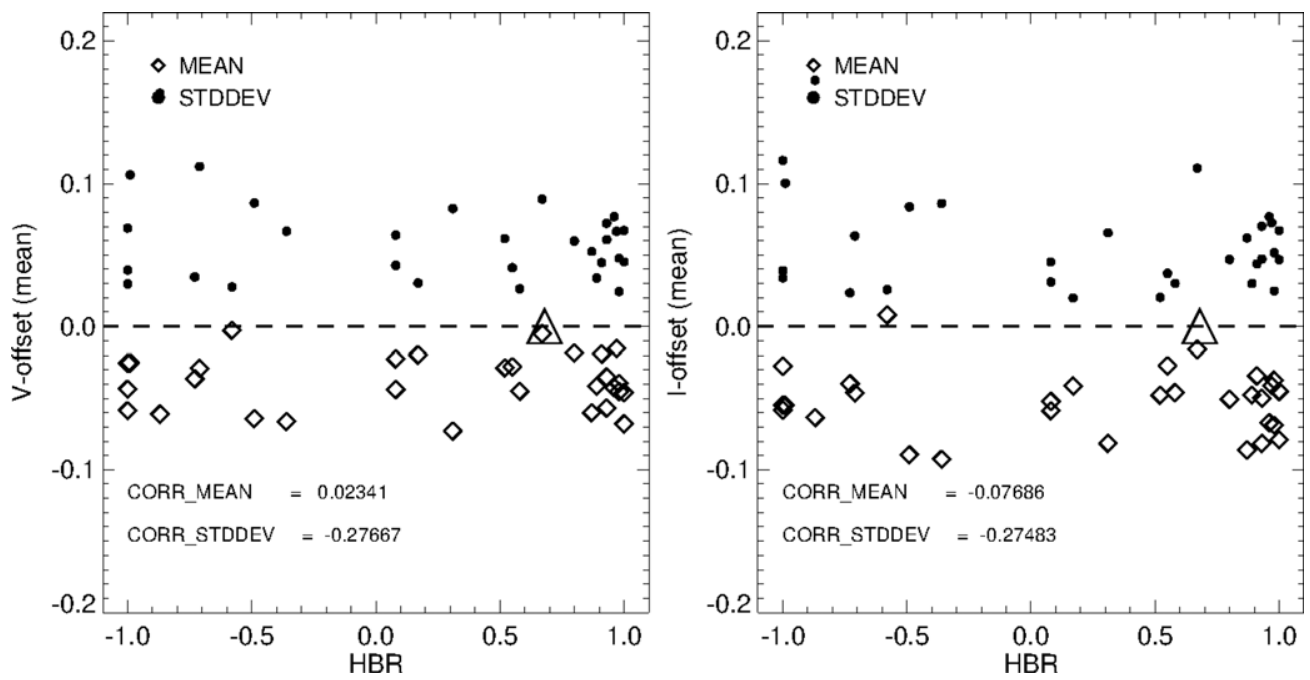

Figure 10. Photometric offset in the $V$ and $I$ bands as a function of the horizontal branch ratio (Harris 1996, on-line edition 2003).

HB structure. To do so we use the HB ratio (HBR) calculated as $\mathrm{HBR}=(B-R) /(B+V+R)$ from Harris (1996; Figure 10). In this formulation, $B$ and $R$ denote the numbers of stars on the blue or red side of the RR Lyrae gap, whereas $V$ represents the number of variables on the HB (Zinn 1986; Lee 1990).
Although the quantification of the HB structure via the HBR is a valuable parameter, in the case of very blue (e.g., NGC 0288 , NGC 6341) or very red (e.g., NGC 0362) HBs, the HBR can become insensitive to the HB morphology (Catelan et al. 2001, and references therein). Therefore we repeat the correlation test 

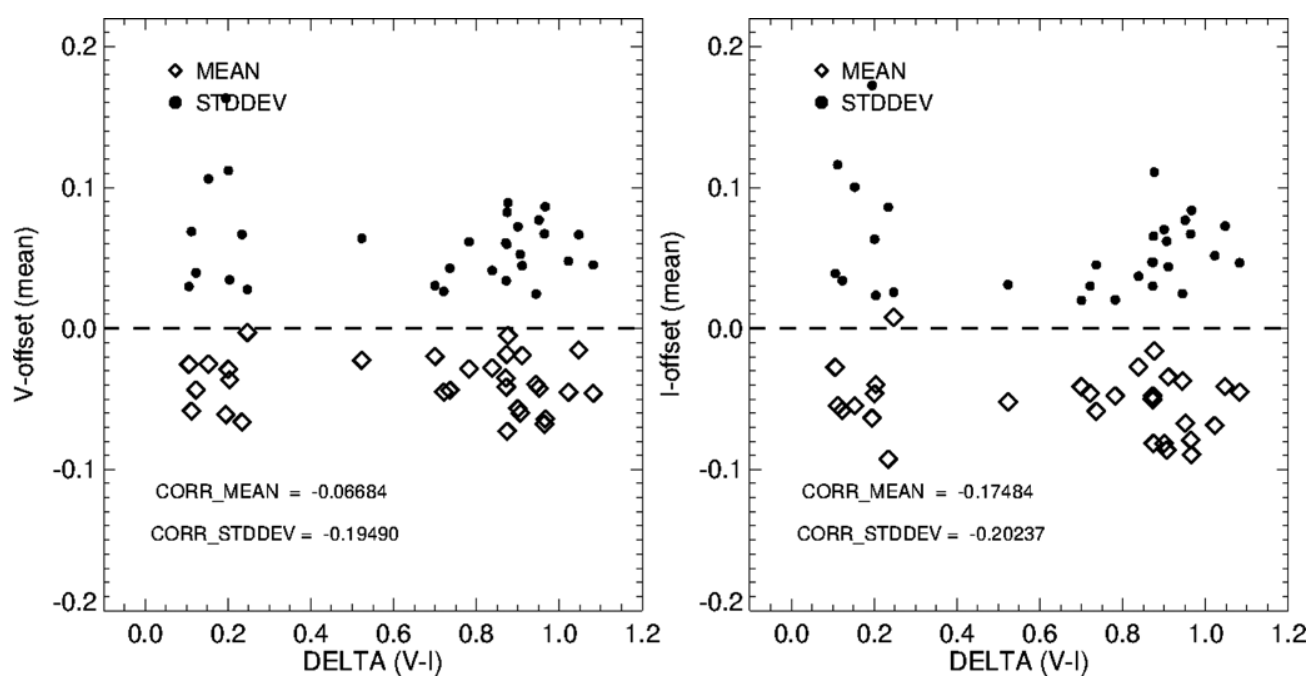

Figure 11. Photometric offset in the $V$ and $I$ bands as a function of the median color difference between HB and the red giant branch (RGB; Dotter et al. 2010).
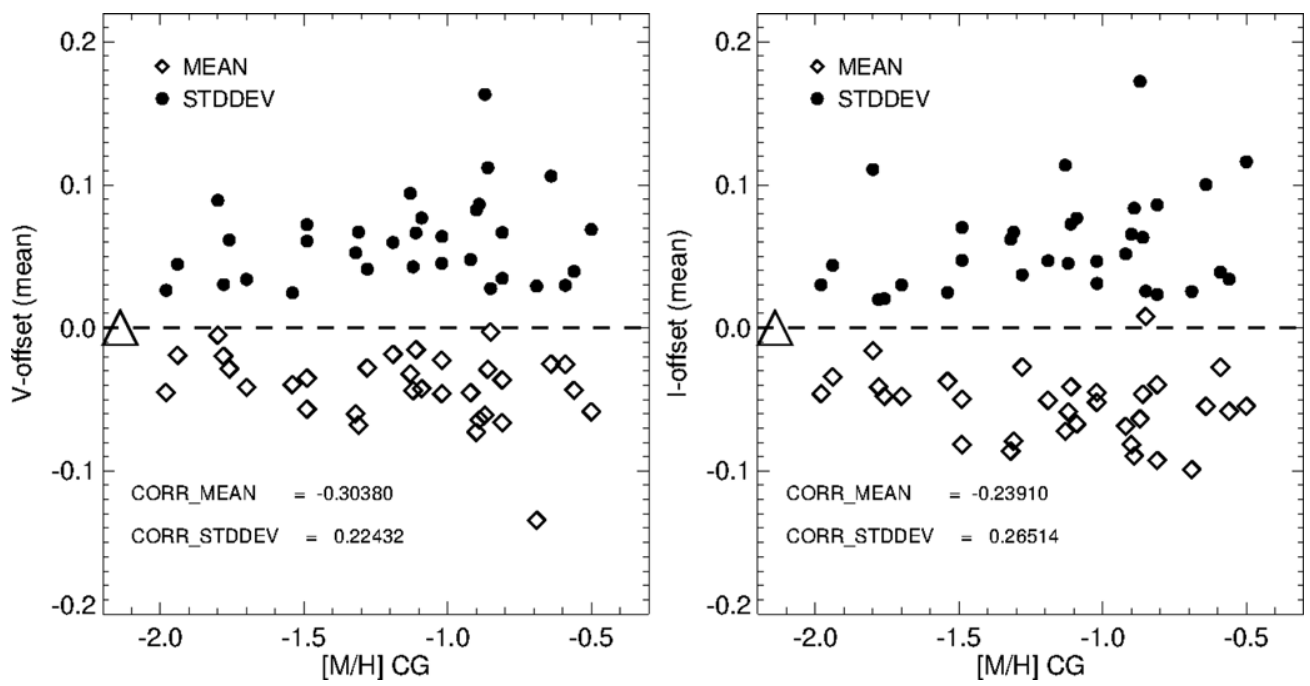

Figure 12. Photometric offset in $V$ and $I$ band (offset $=$ ACS - Stetson) as a function of the globular cluster metallicity. All symbols and colors are as in Figure 7 . The cluster with the largest offset in the $V$-band, at $[\mathrm{M} / \mathrm{H}]=-0.7$, is E3.

and in Figure 11 show the median $(V-I)$ color difference between the HB and the red giant branch (RGB) $\Delta(V-I)$ (Dotter et al. 2010). The correlation coefficients in both cases are in the range of 0.02 and 0.17 , and can therefore be considered to be negligible.

\subsubsection{Metallicity}

As stated by Sirianni et al. (2005), transforming accurately between ACS filters and ground-based filters can be complicated, with potential dependencies on the stellar spectrum, metallicity, and other stellar parameters. The metallicity is likely to be important, but not the only factor playing a role in the filter transformation equations. It is also one of the main parameters affecting the integrated cluster color (see Section 3.2.1), as well as the HB structure (see previous section). The clusters in our sample have metallicities ranging from $[\mathrm{M} / \mathrm{H}]=-1.98(\mathrm{NGC} 5466)$ up to $[\mathrm{M} / \mathrm{H}]=-0.50$ (NGC 5927), excluding NGC 6441 with $[\mathrm{Fe} / \mathrm{H}]=-0.46$. In Figure 12 we show the difference in the two filter offsets, $V$ and $I$, as a function of the metallicity for each GC. The global metallicities $[\mathrm{M} / \mathrm{H}]$ were taken from Marín-Franch et al. (2009) and calculated from $[\mathrm{Fe} / \mathrm{H}]$ iron abundances using the prescrip- tion by Salaris et al. (1993). Based on the correlation coefficients as given in Tables 3 and 4, we conclude that there is a small if any correlation between the $V$ - and $I$-band photometric offsets and the cluster metal abundance.

\subsubsection{Mass}

Recent studies have shown that galactic GCs are not single stellar populations in the strictest sense, a fact first established for the most massive GCs. Although two of the more prominent cases, NGC 1851 and NGC 5139, are also part of this study, it should be noted that NGC 2419 is also among the more massive clusters in the Milky Way and has indeed been found to host a second generation of He-enriched stars (Di Criscienzo et al. 2011). We also note that the value for the NGC 2419 mass, given by Gnedin \& Ostriker (1997), $1.6 \times 10^{6} M_{\odot}$, differs significantly (up to $\sim 50 \%$ ) from the values published by Brüns \& Kroupa (2011), and references therein, which range from 0.9 to $1.19 \times 10^{6} M_{\odot}$ therefore placing the target clusters in relative context to NGC 2419 is difficult. However, in Figure 13 we show the photometric offset in the two filter bands as a function of the total cluster mass (Gnedin \& Ostriker 1997 and references therein). 

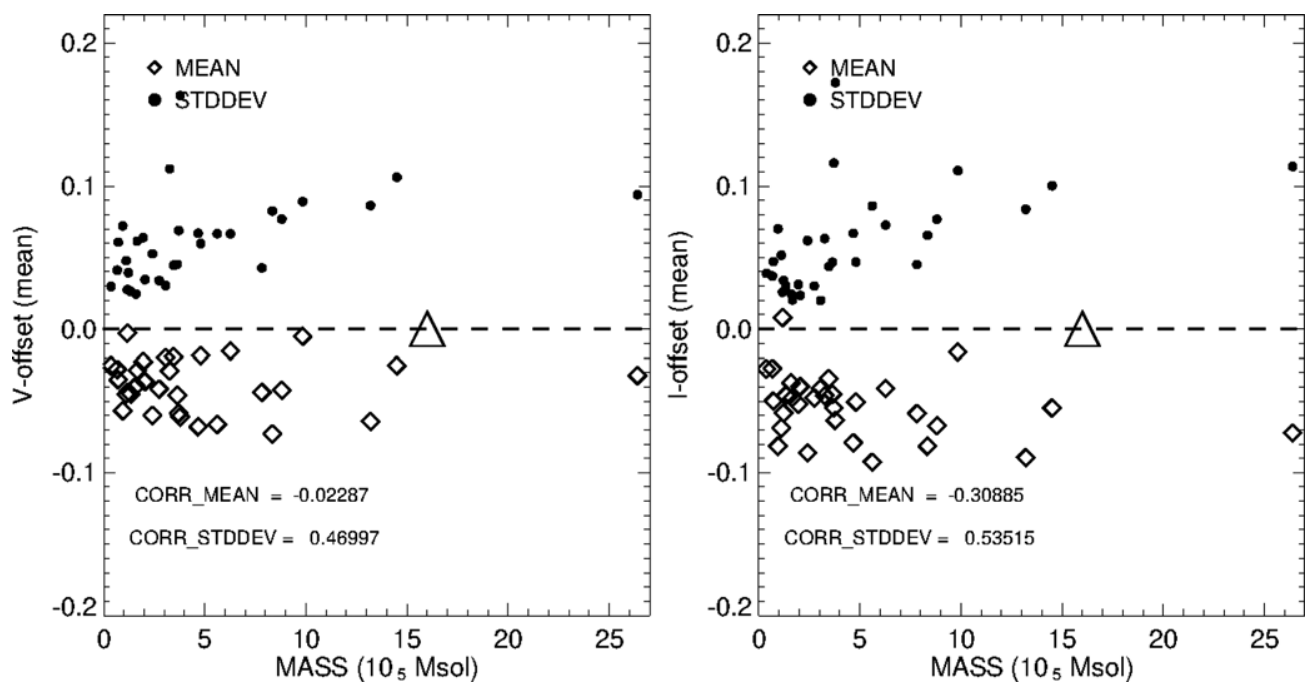

Figure 13. Photometric offset in the $V$ and $I$ bands as a function of the globular cluster mass (Gnedin \& Ostriker 1997).

The correlation coefficients suggest that there is no statistically significant correlation between the photometric offsets and the masses of the GCs. There is a slight tendency for more massive clusters to exhibit a greater dispersion in their magnitude offsets compared with lower mass clusters; however, this could also be a result of the possibility that the photometry of more massive clusters is more likely to be subject to the effects of crowding than that of less massive clusters.

\section{EXTREME CASES OF PHOTOMETRIC OFFSETS}

Our photometric sample is very diverse. Some clusters contain only RGB stars (e.g., NGC 0104, NGC 5139, NGC 6093), whereas others include main sequence (MS), sub-giant branch (SGB), RGB as well as HB stars (e.g., NGC 0288, NGC 5904, NGC 6341). The extreme cases, showing the largest discrepancy between the observed and transformed photometry, or the largest spread $\sigma$ in photometric offset, are hence of special interest. The largest offset in both the $V$ - and $I$-bands is found for the cluster E3. Based on the CMD (see Figure 1), the E3 sample may include field stars and hence not represent a single metallicity, used in the correlation test, i.e., although not being cluster stars but assumed to have the E 3 metallicity, integrated color and HBR. If those stars are indeed contaminants and not cluster stars including them in the analysis will affect the mean offset and consequentially the results of the correlation tests. This may also be true for several other target clusters, not due to contamination by field stars, but because they do not follow the "single stellar population" paradigm as shown by Piotto (2009), and references therein. In our sample these are NGC 0104 (Anderson et al. 2009; Di Criscienzo et al. 2010), NGC 0288 (Piotto et al. 2007; Roh et al. 2011), NGC 1851 (Han et al. 2009), and NGC 5139 (e.g., Lee et al. 1999; Pancino et al. 2000; Bedin et al. 2004; Bellini et al. 2010; see also Figure 14), which all have been found to host multiple MS, SGB, and/or RGB populations. However, in our analysis here the respective stellar samples are too small to show a significant effect, e.g., an increased spread in the photometric offset.

Figure 14 shows the CMD of NGC 5139. Of the 19 stars with both ACS and Stetson photometry, only three may belong to a slightly redder RGB, i.e., be of higher metallicity than the bulk

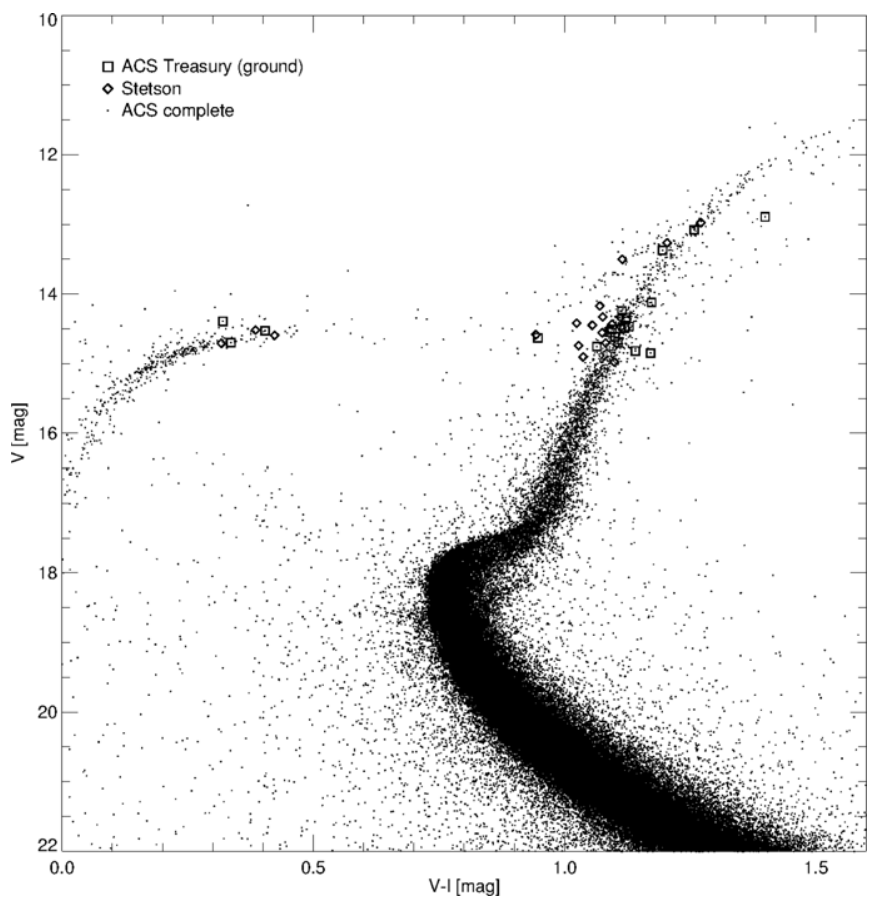

Figure 14. $V$ vs. $(V-I)$ CMD for NGC 5139, showing the converted ACS photometry as open squares, the Stetson data as open diamonds, and the complete ACS sample as dots. Out of 49 stars with matched ACS and Stetson photometry only three could tentatively (if at all) be assigned to a different RGB then the bulk of the RGB stars. This is likely not sufficient to have any effect on the correlation between metallicity and offset.

of the RGB stars. However, only one of those stars shows a photometric offset of $\sim-0.3$ mag in the $I$ band, which does not affect the mean offset or its standard deviation significantly.

The largest dispersion in photometric offset (see Table 2) is found in a different cluster, NGC 0362 (with a sample containing 100 stars). This is somewhat unexpected given that NGC 0362 has a very well defined CMD, with no apparent contaminants. Comparing its properties with the other clusters of our sample, NGC 0362 is by no means an exceptional cluster. Nevertheless, NGC 0362 (in combination with NGC 0288) has been the subject of many studies (Stetson et al. 1996, and references therein), due to their different HB morphologies despite similar 
metallicities and $\alpha$-abundances. The question remains as to why NGC 0362 displays a typical photometric zeropoint offset but an unusually large standard deviation.

With respect to the minimum discrepancy between transformed and observed $V$ and $I$ band, the situation is less clear. NGC 6362, one of the more metal-rich MWGCs $([\mathrm{M} / \mathrm{H}]=$ $-0.85)$ shows the smallest photometric offsets in both filters ( $V$ band: $-0.0028 / 0.0090, I$ band: $0.0080 / 0.0060$ for mean/median offset). However, the NGC 6362 sample contains only 14 stars, which makes this a less solid result compared with the NGC 6205 sample, containing 51 stars. NGC 5053 with 27 stars agrees very well in the $I$ band, and is with respect to the CMD very similar to NGC 2419, with a populated RGB and HB in the combined ACS and Stetson sample.

The available data set shows clearly that the filter transformation based on NGC 2419 alone indeed introduces a systematic offset between the transformed $V$ - and $I$-band magnitudes and their observed counterparts. The correlation between the offset and various cluster parameters causes deviations between the observed and transformed magnitudes of up to $0.3 \mathrm{mag}$, depending on the filter band.

\section{SUMMARY}

The transformation equations traditionally used to convert the ACS F606W and F814W filters of the Wide-Field Camera into ground-based Johnson-Cousins $V$ - and $I$-band magnitudes are based on observations of NGC 2419, one of the most metalpoor and most massive GCs in the Milky Way (Harris 1996, and references therein). In our study we confirm the existence of systematic offsets, expected due to the differences in stellar spectral energy distributions, in the converted ACS photometry and their "original" ground-based counterparts. The latter can, in extreme cases (E 3), reach up to $\gtrsim 0.3 \mathrm{mag}$. However, there are no statistically significant correlations between GC properties, such as metallicity, and the difference between the transformed and observed magnitudes. To the extent that they exist, these correlations seem to be greater in the $V$ band than in $I$. The strongest correlation has been found between the photometric offset and the integrated cluster color, followed by metallicity and total mass.

The correlation between the photometric offset and the total cluster mass is less clear and varies between the two filters. We note as well, that the integrated GC colors (Harris 1996), as well as their masses (Gnedin \& Ostriker 1997, and references therein) are a compilation of different sources, and hence are less uniform.

The transformation equations by Sirianni et al. (2005) are based on the observations of 30-60 NGC 2419 stars, depending on the filter band. Here we provide a database of combined ACS and ground-based optical photometry ( $V$ and $I$ band), which in some cases (e.g., NGC 6341) included several hundreds of stars, populating the whole CMD. However, as seen in Figure 1, not only do the number of stars vary widely, but so does the relative coverage of the CMD. NGC 0288, NGC 5904, and NGC 6341 have the largest number of stars with combined ACS and groundbased photometry, as well as the widest range of evolutionary stages, including MS, SGB, RGB, and HB. NGC 6093, which includes only two stars on the RGB, has been excluded from the correlation analysis. Additional tests have shown that if we restrict our sample to only stars above the MSTO, and hence mimicking the NGC 2419 sample used by Sirianni et al. (2005) more closely, the various linear correlations between the photometric offset and the cluster parameters are unchanged. In a separate series of correlation tests, we excluded NGC 5139 from the cluster sample, given that this MWGC is known to host various stellar populations (see Piotto 2009 and references therein), featuring partly different metallicities. However, the only correlation that is significantly affected is the one between mass and the photometric offset. This is not surprising, given that NGC 5139 is also by far the most massive cluster in our sample, and as a result defines the correlation at the high mass end. In all other correlation tests, rejecting NGC 5139 changed the results insignificantly.

As described in Section 3 we exclude saturated stars from the analysis presented here. However, the correlation test for an unselected sample, i.e., without rejecting saturated stars, finds all correlations to be weaker, with a smaller mean offset, but a larger standard deviation for both filters.

The results of this study show that there are small systematic offsets between transformed ACS and observed ground-based photometry, and that these are only weakly correlated, if at all, with various cluster parameters and their underlying stellar population. As a result, investigators wishing to transform GC photometry from the Sirianni et al. (2005) ground-based $V$, I system onto the Stetson (2000) system simply need to add $-0.040( \pm 0.012)$ to the $V$ magnitudes and $-0.047( \pm 0.011)$ to the $I$ magnitudes. The quoted errors in each case represent the average value of the standard errors of each mean offset. This in turn means that the transformed ACS $V-I$ colors match the ground-based values from Stetson (2000) to within $\sim 0.01 \mathrm{mag}$. We note that these offsets are the average of the mean offset for all clusters in our sample (see also Table 2 ). In contrast to that the results in Section 3.1 are the mean offset for all individual stars, excluding E 3 and NGC 6441. However, within the photometric errors the average and mean offset are in agreement.

Support for this work (proposal GO-10775) was provided by NASA through a grant from the Space Telescope Science Institute, which is operated by the Association of Universities for Research in Astronomy, Inc. under NASA contract NAS526555. Support for M.H. has been provided by the BASAL Center for Astrophysics and Associated Technologies PFB06, the FONDAP Center for Astrophysics No. 15010003, and the Ministry for the Economy, Development and Tourism's Programa Iniciativa Científica Milenio through grant P07-021F, awarded to The Milky Way Millennium Nucleus. S.R.M. was funded by NSF grant AST-0807945. This research used the facilities of the Canadian Astronomy Data Centre operated by the National Research Council of Canada with the support of the Canadian Space Agency.

\section{REFERENCES}

Anderson, J., Piotto, G., King, I. R., Bedin, L. R., \& Guhathakurta, P. 2009, ApJL, 697, L58

Anderson, J., Sarajedini, A., Bedin, L. R., et al. 2008, AJ, 135, 2055

Barmby, P., Huchra, J. P., Brodie, J. P., et al. 2000, AJ, 119, 727

Baumgardt, H., Côté, P., Hilker, M., et al. 2009, MNRAS, 396, 2051

Bedin, L. R., Piotto, G., Anderson, J., Cassisi, S., \& King, I. R. 2004, ApJ, 605,125

Bellini, A., Bedin, L. R., Piotto, G., et al. 2010, AJ, 140, 631

Brüns, R. C., \& Kroupa, P. 2011, ApJ, 729, 69

Cardelli, J. A., Clayton, G. C., \& Mathis, J. S. 1989, ApJ, 345, 245

Carretta, E., \& Gratton, R. G. 1997, A\&AS, 121, 95

Catelan, M., Bellazzini, M., Landsman, W. B., et al. 2001, AJ, 122, 3171

Côté, P., Blakeslee, J. P., Ferrarese, L., Jordán, A., \& Mei, S. 2004, ApJS, 153,223

Da Costa, G. S., Held, E. V., Saviane, I., \& Gullieuszik, M. 2009, ApJ, 705,1481 
Di Criscienzo, M., D’Antona, F., Milone, A. P., et al. 2011, MNRAS, 414, 3381

Di Criscienzo, M., Ventura, P., D’Antona, F., Milone, A., \& Piotto, G. 2010, MNRAS, 408, 999

Dotter, A., Sarajedini, A., Anderson, J., Aparicio, A., \& Bedin, L. R. 2010, ApJ, 708,698

Gilliland, R. 2004, ACS/ISR 2004-01, CCD Gains, Full Well Depths, and Linearity up to and Beyond Saturation

Gnedin, O. Y., \& Ostriker, J. P. 1997, ApJ, 474, 223

Han, S.-I., Lee, Y.-W., Joo, S.-J., Sohn, S. T., \& Yoon, S.-J. 2009, ApJ, 707, 190

Harris, W. E. 1996, AJ, 112, 1487 (2010 edition)

Jordán, A., Blakeslee, J. P., Côté, P., Ferrarese, L., \& Infante, L. 2007, ApJS, 169,213

Lee, Y.-W. 1990, ApJ, 363, 159

Lee, Y.-W., Joo, J.-M., Sohn, Y.-J., et al. 1999, Natur, 402, 55

Marín-Franch, A., Aparicio, A., Piotto, G., et al. 2009, ApJ, 694, 1498

Milone, A. P., Piotto, G., Bedin, L. R., et al. 2010, in SF2A-2010: Proc. Annual Meeting of the French Society of Astronomy and Astrophysics, ed. S. Boissier, M. Heydari-Malayeri, R. Samadi, \& D. Valls-Gabaud, 319

Pancino, E., Ferraro, F. R., Bellazzini, M., Piotto, G., \& Zoccali, M. 2000, ApJL, 534, L83

Pavlovsky, C., Koekemoer, A., \& Mack, J. 2005, ACS Data Handbook, Version 4.0 (Baltimore, MD: STScI)
Piotto, G. 2009, in IAU Symp. 258, The Ages of Stars (Cambridge: Cambridge Univ. Press), 233

Piotto, G., Bedin, L. R., Anderson, J., King, I. R., \& Cassisi, S. 2007, ApJ, 661,53

Roh, D.-G., Lee, Y.-W., Joo, S.-J., et al. 2011, ApJ, 733, 45

Saha, A., Dolphin, A. E., Thim, F., \& Whitmore, B. 2005, PASP, 117, 37

Salaris, M., Chieffi, A., \& Straniero, O. 1993, ApJ, 414, 580

Sarajedini, A., Bedin, L. R., Chaboyer, B., Dotter, A., \& Siegel, M. 2007, AJ, 133,1658

Sirianni, M., Jee, M. J., Benítez, N., Blakeslee, J. P., \& Martel, A. R. 2005, PASP, 117,1049

Stetson, P., VandenBerg, D. A., \& Bolte, M. 1996, PASP, 108, 560

Stetson, P. B. 2000, PASP, 112, 925

Stetson, P. B. 2005, PASP, 117, 563

Suntzeff, N. B., Kraft, R. P., \& Kinman, T. D. 1988, AJ, 95, 91

Taylor, M. B. 2005, in ASP Conf. Ser. 347, Astronomical Data Analysis Software and Systems XIV, ed. P. Shopbell, M. Britton, \& R. Ebert (San Francisco, CA: ASP), 29

Zinn, R. 1985, ApJ, 293, 424

Zinn, R. 1986, in Proc. Meeting on Stellar Populations, Baltimore, MD, 1986 May 20-22, ed. C. A. Norman, A. Renzini, \& M. Tosi (Cambridge: Cambridge Univ. Press), 73 